\title{
El gran impacto del Metro
}

Louis DE GRANGE C. Profesor de la Escuela de Ingeniería Industrial de la Universidad Diego Portales.

RESUMEN | Las grandes ciudades del mundo que han resuelto su problema de transporte público lo han logrado principalmente a la luz de las siguientes dos políticas públicas: una expansión significativa de la red de Metro y trenes urbanos, y una firme regulación en el uso del automóvil. La evidencia empírica a nivel mundial ha permitido constatar que la expansión de redes de Metro genera una reducción mucho más efectiva en el uso del transporte privado que otras medidas como por ejemplo subsidiar la tarifa del transporte público. Adicionalmente, la existencia de una importante red de Metro o trenes urbanos permite aumentar notablemente la efectividad de políticas regulatorias al uso del automóvil, como tarificación vial y gravámenes específicos, entre otras, ya que los automovilistas habituales sí ven en el Metro una alternativa real de transporte, no así en los servicios de buses.

PALABRAS ClAVE | Áreas metropolitanas, modelos de transporte, movilidad, planificación urbana, transporte urbano.

ABSTRACT | Large cities around the world that have successfully addressed their public transport problems have made it mainly through the following two public policies: a significant expansion of the Metro or urban train networks, and a tight regulation of the use of automobiles. Empirical evidence worldwide shows that the expansion of the Metro network produces a much more effective reduction in the use of private transport than alternative policies as for instance subsidizing the tariff of public transport. Besides, the existence of an important Metro or urban train network enlarges the effectiveness of other regulatory policies to the use of automobile, as road pricing and specific taxes, since the users of automobiles see in Metro a reasonable alternative of transport, which is not the case for buses.

KEY WORDS | Metropolitan areas, transportation models, mobility, urban planning, urban transportation.

Correspondencia: E-mail: louis.degrange@udp.cl 
No existe ninguna gran ciudad en el mundo, de tamaño y población comparable con la de Santiago, que haya resuelto el problema de transporte público con buses. Ciudades como Londres, Madrid, París, Berlín, Hong Kong, Barcelona, Nueva York, Tokio, entre muchas otras, cuyas extensiones urbanas y poblaciones son relativamente comparables con la de Santiago, tienen redes de Metro o trenes urbanos que superan los 200, 300 e incluso los 400 kilómetros, cifra hasta cuatro veces superior al caso de nuestra capital. En estas ciudades los buses representan sólo entre el 13\% y el 25\% del total de viajes en transporte público al interior de la urbe; la mayor parte del trabajo es realizado por el Metro o tecnologías similares. Es decir, en ciudades que presentan buenos sistemas de transporte público, la mayor parte de la demanda es atendida por líneas de Metro o tecnologías similares (trenes suburbanos, trenes ligeros o tranvías), siendo el bus una alternativa minoritaria.

Un caso interesante de mencionar es el plan de transporte urbano de San Pablo en Brasil, proyectado para el año 2020. El denominado PITU 2020 considera una inversión de US\$22 mil millones en 284 kilómetros de Metro, US\$900 millones en un tren al aeropuerto y US\$ 1.300 millones en ferrocarriles suburbanos. Es decir, del total de US\$24 mil millones de dólares presupuestados, un $92 \%$ será destinado a nuevas líneas de Metro, y el resto en tecnologías similares para sectores periféricos de la ciudad. $\mathrm{Al}$ respecto, es interesante notar que los brasileños son uno de los principales fabricantes de buses en el mundo, y pese a ello han priorizado la construcción de nuevas líneas de Metro en la ciudad de San Pablo, evidentemente debido a las incomparables ventajas de este modo de transporte en las grandes urbes.

¿Por qué entonces insistir en priorizar los buses en Santiago con un subsidio que supera el $40 \%$ de los costos de provisión de estos servicios? Dejando de lado la contingencia política causada por el desastre inicial del Transantiago implementado en el año 2007, la insistencia con los buses obedece a los mitos que existen, tanto en el mundo académico como político, respecto al elevado costo que significa invertir en trenes urbanos. Al respecto, la literatura especializada ha reconocido que sobre los 15 mil pasajeros por hora un servicio de Metro puede ser más barato que uno de buses. La razón de ello es simplemente que el costo variable por pasajero transportado en el caso del Metro es casi la tercera parte que el de los buses. Luego, aunque el costo de la inversión de Metro sea muy alto, las altas demandas que garantizan los servicios de Metro bien diseñados inducen finalmente un menor costo de provisión del servicio.

De hecho, el pago que ha recibido el Metro de Santiago por cada pasajero transportado durante los años 2008 y 2009 es más bajo que el de cualquier otro operador del Transantiago, promediando los $\$ 280$, mientras que algunos operadores de buses reciben $\$ 400$, $\$ 600$, e incluso sobre los $\$ 800$ por cada pasajero que usa sus servicios. Notar que estas cifras corresponden al período en el cual el Transantiago ya se ha estabilizado, y son las que finalmente obligaron la creación de la ley de subsidio permanente al transporte público.

Todos estos antecedentes, sumados a los que se exponen a continuación, dejan en evidencia lo fundamental que es para los habitantes de Santiago contar con nuevos proyectos, como la anunciada Línea 6 de Metro, así como también la postergada Línea 3 entre Irarrázaval e Independencia. La evidencia a favor de Metro es simplemente contundente.

\section{El impacto del metro y trenes urbanos en el mundo, ¿qué dice la lite- ratura especializada?}

Los trabajos empíricos respecto al impacto que el Metro o tecnologías similares generan sobre las ciudades y la calidad de vida de sus habitantes son bastante amplios. A continuación se exponen aquellos trabajos más recientes respecto a este tema. 
En el artículo de Partridge et al. (2007) se muestra el impacto que tiene el Metro sobre la cantidad de puestos de trabajo en las ciudades de Estados Unidos. Específicamente, se obtiene que el cambio porcentual en la cantidad de empleos entre los años 1990 y 2004, para ciudades sin líneas de Metro, fue de 18,1\%, mientras que en ciudades con Metro el cambio fue de $35,8 \%$. También se demuestra en este trabajo que la distancia media de viaje en ciudades con Metro tiende a reducirse con el tiempo, debido a la relocalización de actividades como comercio, servicios y vivienda en torno a los proyectos de Metro. Este cambio en las localizaciones en torno al Metro se traduce en menores costos de combustible, menores tiempos de viaje y menos externalidades como contaminación y congestión.

También se ha demostrado (Baum-Snow y Kahn, 2005) que la expansión de los trenes urbanos o Metro generan cambios en la localización de actividades, modificando usos de suelos de tal forma que se reduce la distancia media de los viajes en automóvil, generando beneficios por menor contaminación y congestión (es decir, más Metro genera viajes más cortos en auto, aunque los usuarios de auto no usen el Metro; esto, debido al impacto del Metro en el sistema de actividades).

Por otra parte, se ha verificado en promedio (Vuk, 2005) que de los usuarios de un nuevo proyecto de Metro, un $70 \%$ proviene de los buses, un 15\% son antiguos usuarios del automóvil y un $15 \%$ es nueva demanda, es decir, viajes que antes de existir el Metro simplemente no se realizaban. En el trabajo de Knowles (1996) se estimó que la demanda inducida por un tren ligero en Manchester fue superior al 20\%, mientras que Monzón (2000) estima una demanda inducida de un 25\% producto de una nueva línea de Metro en Madrid. En el Metro de Atenas se detectó que un 16\% de los usuarios provenía del automóvil (Golias, 2002), mientras que en el caso de Madrid la cifra se estimó en un 26\% en el caso de un tren suburbano (Monzón, 2000). En síntesis, más Metro significa más viajes, más actividades y más crecimiento.

En el trabajo de Litman (2009) se presenta una serie de interesantes comparaciones de ciudades de Estados Unidos con y sin líneas de Metro o Trenes Ligeros (LRT). Para efectuar el análisis comparado, Litman (2009) define tres tipos de ciudades: a) Large Rail (LR): ciudades en que el Metro o los LRT representan más del 50\% de los viajes en transporte público; b) Small Rail (SR): ciudades en que el Metro o los LRT representan menos del 50\% de los viajes en transporte público; c) Bus Only (BO): ciudades en que no tienen ni Metro ni LRT.

Comparando las ciudades tipo BO con las ciudades tipo LR, se constató que estas últimas presentan mucho mejor nivel de servicio en lo que es transporte público, además de una importante superioridad en muchas otras variables que afectan la calidad de vida de las ciudades. Específicamente, es interesante destacar los siguientes resultados:

- En las ciudades LR los viajes per cápita en transporte público son un 400\% mayor que en ciudades $\mathrm{BO}$. Es decir, se realizan 5 veces más viajes en transporte público debido a la presencia de Metro.

- El total de muertos per cápita en accidentes de tránsito en ciudades LR es un 24\% menos que en ciudades SR, y un 36\% menos que en ciudades BO.

- La cantidad de vehículos-kilómetro per cápita en las ciudades LR es un 13\% menor que en las SR, y un $21 \%$ menor que en las ciudades BO. Respecto a la tasa de motorización, en el caso de las ciudades $\mathrm{LR}$ es 0,68 , en las $\mathrm{SR}$ es 0,77 y en las $\mathrm{BO}$ es 0,80. Esto es especialmente interesante, ya que el ingreso per cápita en las ciudades LR es generalmente mayor que en las otras. 
- En ciudades BO y SR, los costos de congestión tienden a crecer conforme aumenta el tamaño de la ciudad. Sin embargo, este patrón no se presenta en las ciudades.

- En las ciudades LR, los beneficios sociales que producen los sistemas de Metro son largamente superiores que los costos y subsidios requeridos para su implementación.

- En ciudades de mayor tamaño y, por lo tanto, con mayor congestión, el costo por pasajero-kilómetro en bus tiende a crecer debido a la congestión, mientras que en el caso del Metro, el costo por pasajero-kilómetro tiende a decrecer con el tamaño de la ciudad debido a las fuertes economías de escala del Metro.

- Respecto al nivel de emisión de contaminantes per cápita, en el caso de las ciudades LR es significativamente menor que en las ciudades BO.

En el trabajo de Agostini y Palmucci (2008a) se analiza el efecto que han tenido las Líneas 1,2 y 5 de Metro sobre el precio de las viviendas en la ciudad de Santiago de Chile. Mediante un enfoque de precios hedónicos (comparación de precios de viviendas de similares características pero con y sin disponibilidad de Metro), las viviendas localizadas en el área de influencia del Metro presentan un mayor valor promedio (respecto de aquellas que se ubican fuera del área de influencia), de 8,84\%, 27,16\% y 6,72\% para las Líneas 1, 2 y 5 respectivamente. En la comuna de Providencia, las viviendas dentro del área de influencia de la Línea 1 y 5 muestran un mayor valor del orden de $12,2 \%$ y $4,1 \%$, respectivamente, mientras que en la comuna de Santiago las viviendas localizadas dentro del área de influencia de las Líneas 1, 2 y 5 del Metro presentan un mayor valor del orden de $16,9 \%, 10,2 \%$ y $11,6 \%$, respectivamente.

De este trabajo también se desprende que la capitalización del valor del Metro en el precio de las viviendas puede producir un aumento en la recaudación por contribuciones pagadas. Este revalúo generado por una línea de Metro puede significar hasta el 20\% de la inversión de la Línea de Metro, tal como se estimó para la Línea 4 de Metro a Puente Alto (ver Agostini y Palmucci, 2008b).

En esta misma línea investigativa, Smith y Gihring (2003) demuestran que, aunque el sistema de Metro tiene mayores costos de inversión inicial, una parte importante de dichos costos son compensados por el mayor valor de las propiedades, mayor productividad y mayor comercio en torno a las estaciones, algo que no ocurre en el caso de los corredores de buses.

En el trabajo de Debrezion et al. (2007) se obtiene, mediante un análisis empírico, que las propiedades cercanas a estaciones de Metro (menos de 400 metros) aumentan su valor en un $14,1 \%$ en promedio, mientras que las propiedades cercanas (menos de 400 metros) a corredores de buses o BRT bajan su valor un 1,3\% en promedio, aunque este último caso no es estadísticamente fuerte, por lo que podría argumentarse que los corredores de buses no impactan el valor de las propiedades. Este último punto es de gran importancia en las políticas públicas, ya que una metodología estándar de evaluación socioeconómica de proyectos de transporte se basa en estimar los beneficios a partir de las variaciones en los precios de las propiedades, pues representan en términos monetarios las mejoras en accesibilidad al área de influencia del proyecto. Luego, y como se aprecia claramente en el estudio de Debrezion et al. (2007), el Metro tiene impactos muchísimo mayores que los buses, y que se resumen en la variación en los precios de las propiedades.

$\mathrm{Al}$ respecto, podemos tratar de respondernos la siguiente pregunta para el caso de Santiago: ¿cuánto ha aumentado el precio de los terrenos en los corredores de Av. Grecia, Av. Santa Rosa o Av. Pajaritos? ¿Cuántos nuevos proyectos inmobiliarios se han desarrollado en torno a los corredores segregados de buses, y cuántos en torno a las nuevas extensiones de Metro? 
Es posible concluir que el Metro genera importantes cambios en los patrones de demanda, sistemas de actividades y localizaciones de servicios, los cuales tienden a conformar una ciudad más sustentable. Ninguno de estos importantes cambios puede obtenerse con un sistema basado principalmente en buses, como demuestran los trabajos internacionales recién expuestos.

\section{Metro: una alternativa real al uso del automóvil}

Respecto al impacto que genera la ampliación de líneas de Metro sobre la demanda por servicios de transporte público y privado al interior de las ciudades, los resultados indican que esta alternativa de transporte, pese a sus mayores costos de inversión y capital, normalmente logra reducir el aumento progresivo en el uso del automóvil, tendiendo a revertir la tendencia hacia una menor participación del transporte público.

Ningún usuario habitual del automóvil querrá voluntariamente dejar su vehículo para subirse a una micro del Transantiago, pero sí podría pensarlo para subirse al Metro, tal como ha quedado en evidencia con la extensión de la Línea 1 a Los Dominicos, y en muchos otros estudios internacionales. De hecho, las cifras en Santiago indican que los días de dos dígitos de restricción a los vehículos catalíticos en Santiago los viajes diarios en automóvil se reducen en un 6\%, que significa cerca de 200 mil viajes diarios menos en automóvil. Lo notable es que 120 mil de estos se traspasan al Metro (un 60\% de la reducción de viajes en auto), y prácticamente ninguno a los buses.

Por otra parte, y como se expone en el trabajo de Baum-Snow y Kahn (2005), aunque el transporte público ha tendido a decrecer en las últimas décadas conforme aumenta el ingreso per cápita de las ciudades, dicha reducción en ciudades con Metro ha sido entre $20 \%$ y $23 \%$, mientras que en ciudades sin Metro la reducción en el uso del transporte público ha sido superior al $60 \%$.

En esta misma línea, Litman (2005) muestra que en los hogares ubicados en las cercanías de estaciones de Metro la posesión de automóvil es inferior que en sectores sin Metro. Bento et al. (2003) concluye que un aumento en el 10\% de la red de Metro reduce en un 4,2\% el uso del automóvil. Esta reducción en el uso del automóvil es mayor a la generada por cualquier política de tarificación vial hasta ahora implementada en alguna ciudad del mundo.

De acuerdo a Lane (2008), sistemas de transporte masivo como Metro proporcionan una mayor comodidad y rapidez que los buses, y permiten una mejor integración con otros modos de transporte (autos, bicicletas y buses). Además, demuestran que el Metro atrae a más usuarios del auto que los buses y, en general, es más atractivo para aquellos usuarios que realmente tienen la posibilidad de escoger entre modos alternativos de transporte. De hecho, entre los viajeros que disponen de auto, un 64\% declaró al modo Metro como alternativa de transporte, mientras que en el caso de los buses la cifra llegó sólo al 18\%.

En consecuencia, el Metro tiende a reducir los viajes en auto por dos razones: primero, por el mayor traspaso que se observa de usuarios provenientes del automóvil y, segundo, por la reducción en la adquisición de automóviles en los hogares ubicados en sectores cercanos a estaciones de Metro. A esto debe sumarse el efecto de largo plazo que genera Metro en las distancias medias de viaje, que tienden a reducirse producto de la concentración de actividades, disminuyendo con ello el kilometraje de todos los modos de transporte, incluyendo el auto. 


\section{Sustentabilidad y futuro de una ciudad con más Metro}

La metodología desarrollada y utilizada por Mideplan en la evaluación social de las distintas líneas de Metro, y en particular de la recientemente anunciada Línea 6 entre Cerrillos y Providencia, presenta una serie de limitaciones que reduce significativamente la rentabilidad social de este tipo de proyectos.

En primer lugar, no considera la liberación de espacios públicos que genera el Metro, resultado opuesto al que se observa cuando se compara con un corredor segregado de buses. En segundo lugar, el Metro no interfiere con los restantes vehículos que utilizan la red vial (autos, camiones, taxis, otros buses, etc.), como sí lo hacen los corredores segregados, y que en muchos casos pueden perjudicar notoriamente a los restantes vehículos. En tercer lugar, las evaluaciones sociales de proyectos de Metro no consideran los efectos de largo plazo sobre el sistema de actividades y su concentración, resultado que no es posible de obtener con buses. Tampoco se considera la mayor confiabilidad y seguridad de los servicios de buses, ni menor accidentabilidad y mortalidad.

En el trabajo de Winston y Larger (2004) se hace notar que la congestión vial se reduce en ciudades cuya oferta de Metro se expande, pero crece cuando se aumenta la oferta de buses. Esto ocurre, según explican los autores, debido a que los buses compiten con los demás vehículos por la vialidad existente, contribuyen a la congestión vial y tienen un bajo impacto en la accesibilidad. En ciudades que han implementado proyectos de Metro, el índice de congestión antes de la implementación del Metro crecía en promedio un 2,8\% anual, mientras que después del Metro dicho índice aumentaba sólo un 1,5\% anual.

Si en la metodología de evaluación social de proyectos de Metro se incluyeran estos beneficios adicionales, además de los gigantescos ahorros de tiempo, descongestión, descontaminación y muchos otros que no alcanzo a detallar en este espacio, sería fácil demostrar que la postergación de otros proyectos de Metro, como la reconocida Línea 3 por Irarrázaval e Independencia, se traduce finalmente en un tremendo costo para la sociedad.

El Metro permite contribuir eficazmente al logro de todos los objetivos de una política de desarrollo urbano: mejora la eficiencia de la economía de la ciudad al reducir los costos de viajar, y además genera un mayor nivel de actividades en el centro de la ciudad aprovechando las economías de aglomeración. El impacto de la pobreza puede ser reducido directamente, donde Metro es el principal transporte de los más pobres, e indirectamente a través del beneficio que los más pobres reciben de la prosperidad económica. También puede mejorar la sustentabilidad, incentivando una reducción voluntaria en el uso del automóvil, y en el mediano plazo por medio del apoyo a una estructura de uso de suelo ambientalmente más favorable.

Todos estos aspectos deben obligadamente ser considerados antes de comparar al Metro con otras alternativas de transporte público masivo. De lo contrario, seguiremos postergando proyectos de Metro que son socialmente rentables. IEURE

\section{Referencias bibliográficas}

Agostini, C. \& Palmucci, G. (2008a). Capitalización Heterogénea de un Bien Semi-Público: El Metro de Santiago. Cuadernos de Economía, 45, 105-128.

Agostini, C. \& Palmucci, G. (2008b). The Anticipated Capitalisation Effect of a New Metro Line on Housing Prices. Fiscal studies, 29, 233-256. 
Baum-Snow, N. \& Kahn, M. (2005). Effects of Urban Rail Transit Expansions: Evidence from Sixteen Cities, 1970-2000. Brookings-Wharton Papers on Urban Affairs, 160.

Bento, A. M., Cropper, M., Mobarak, A. M. \& Vinha, K. (2003). The Impact of Urban Spatial Structure on Travel Demand in the United States. Review of Economics and Statistics, 87, 466-478.

Debrezion, G., Pels, E. \& Rietveld, P. (2007). The Impact of Railway Stations on Residential and Com mercial Property Value: A Meta-analysis. Journal of Real Estate Finance and Economics, 35:161-180.

Golias, J.C. (2002). Analysis of traffic corridor impacts from the introduction of the new Athens Metro system. Journal of Transport Geography 10 (2), 91-97.

Knowles, R. (1996). Transport impacts of Greater Manchester's Metrolink light rail system. Journal of Transport Geography, 4, 1-14.

Lane, B. W. (2008). Significant Characteristics of The Urban Rail Renaissance In The United States: A Discriminant Analysis. Transportation Research, 42A, 279-295.

Litman, T. (2005), Land Use Impacts on Transportation. Victoria Transport Policy Institute (www.vtpi. org); at www.vtpi.org/landtravel.pdf

Litman, T. (2009). Rail Transit In America: A Comprehensive Evaluation of Benefits. Victoria Transport Policy Institute. http://www.vtpi.org/railben.pdf

Monzón, A. (2000). Travel demand impacts of a new privately operated suburban rail in the Madrid N-III corridor. European Transport Research Conference. 7-11 Sept. Cambridge, UK.

Partridge, M. D., Rickman, S., Ali, K. \& Olfert, M. R. (2007). The Landscape of Urban Influence on U.S. County Job Growth. Review of Agricultural Economics, 29, 381-389.

Smith, J. J. \& Gihring, T. A. (2003). Financing Transit Systems Through Value Capture: An Annotated Bibliography. Geonomy Society. www.progress.org/geonomy at www.vtpi.org/smith.pdf

Vuk, G. (2005). Transport impacts of the Copenhagen Metro. Journal of Transport Geography, 13, 223-233.

Winston, C. and Langer, A. (2004), The Effect of Government Highway Spending on Road Users' Congestion Costs. Brookings Institute. www.brookings.edu 